\title{
Figures de l'excès dans Antony and Cleopatra
}

Antony and Cleopatra, a tragedy of excess

\section{François Laroque}

\section{(2) OpenEdition \\ Journals}

\section{Édition électronique}

URL : http://journals.openedition.org/shakespeare/1061

DOI : 10.4000/shakespeare.1061

ISSN : 2271-6424

Éditeur

Société Française Shakespeare

Édition imprimée

Date de publication : 1 novembre 2007

Pagination : 103-118

ISBN : 2-9521475-4-X

Référence électronique

François Laroque, "Figures de l'excès dans Antony and Cleopatra », Actes des congrès de la Société française Shakespeare [En ligne], 25 | 2007, mis en ligne le 10 février 2008, consulté le 01 mai 2019. URL : http://journals.openedition.org/shakespeare/1061 ; DOI : 10.4000/shakespeare.1061 


\section{Shakespeare et l'excès}

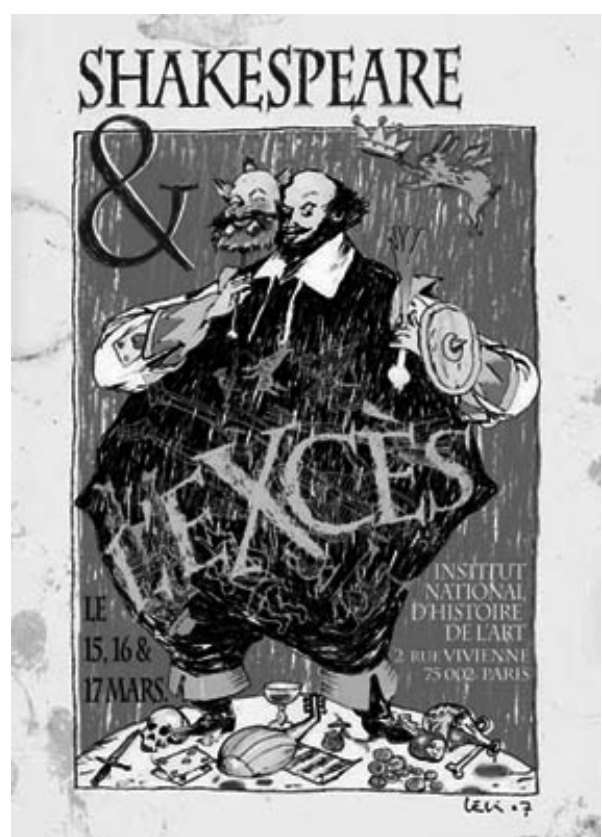

actes du Congrès

organisé par la

SOCIÉTÉ FRANÇAISE SHAKESPEARE

les 15,16 et 17 mars 2007

textes réunis par

Pierre KAPITANIAK

sous la direction de

Jean-Michel DÉPRATS 
COUVERTURE :

Edouard Lekston, Falstaff, 2007

conception graphique et logo

Pierre Kapitaniak

\section{(C) 2007 Société Française Shakespeare \\ Institut du Monde Anglophone}

Université de Paris III - Sorbonne Nouvelle

5 rue de l'École de Médecine 75006 Paris

www.societefrancaiseshakespeare.org

Tous droits de traduction, de reproduction et d'adaptation réservés pour tous les pays 
FIGURES DE L'EXCÈS DANS ANTONY AND CLEOPATRA

François LAROQUE

\begin{abstract}
Contrairement à Titus Andronicus, où l'excès s'alimente directement à la source d'une rhétorique hypertrophiée (celle des drames de Sénèque) et confine au grotesque et au grand guignol, Antoine et Cléopâtre prend soin de mesurer l'écart ou l'outrance en les mettant constamment en perspective grâce aux jeux d'oppositions entre la tempérance romaine et la surenchère égyptienne. La pièce met en abyme les deux figures centrales du paradoxe et de l'hyperbole respectivement incarnées par Cléopâtre et Marc Antoine sur la scène du monde (" the three-nooked world "), où l'influence de Tamburlaine est marquée, pour les opposer à une stratégie de la réticence et du calcul. Comme dans la moralité tudor, le combat amoureux et politique s'inscrit ici dans le cadre d'un débat qui oppose les valeurs et les croyances de deux civilisations. Dès lors la dynamique de l'œuvre met en place un système de vases communicants où l'Être (la figure de la plénitude dont la marque est la réussite) oscille entre des pôles contraires avant que les coefficients de départ ne s'inversent, faisant ainsi de l'exagération, de l'outrance ou de l'impossible, non plus la toile de fond de la caricature grivoise ou misogyne mais les principes d'un nouveau mode d'être: "His legs bestrid the ocean " (v.ii.81). Dans ce que H.W. Fawkner appelle "l'hyperontologie" de la pièce la folie, la perte ou la débauche se retournent en leurs contraires et deviennent synonymes de la sérénité, du gain et de l'abondance retrouvés.
\end{abstract}

Antony and Cleopatra, a tragedy of excess Contrary to Titus Andronicus, where excess directly results from the hyperbolic rhetoric of Seneca's tragedies and comes close to grotesque and grand-guignol, Antony and Cleopatra constantly puts exaggeration in perspective thanks to the polarities that pit Roman temperance against Egyptian "o'erflowing". The play, where the influence of Tamburlaine is quite visible, puts in the foreground the two key-figures of paradox and hyperbole, respectively illustrated by the characters of Cleopatra and Mark Antony on the stage of the world ("the three-nooked world"), where they are confronted to a strategy of ellipsis and anticipation. As in the Tudor morality play, the erotic and political struggle is here part of a general debate that contrasts the values and beliefs of a civilization against those of another. The dynamic forces at work in the play are part of a system whereby the figure of fullness that goes along with success is caught between antagonist polarities, before the main data and hierarchies of the initial situation are put upside down, thus turning exaggeration, excess or impossibility the very bases of a new mode of being rather than the tools for bawdy and misogynous caricature: "His legs bestrid the ocean" (v.ii.81). What the critic H.W. Fawkner calls the "hyperontology" of the play allows in fact madness, loss or debauchery to be turned inside out into their own contraries, so that they ultimately become synonymous with the recovery of peace of mind, gain and plenty.

'est sans doute peu de dire qu'une œuvre de l'ampleur d'Antony and Cleopatra ${ }^{141}$ (42 personnages répartis sur cinq actes découpés en 42 scènes) est un formidable embarras de richesses, une œuvre où l'excès est partout. Dans cette tragédie amoureuse qui est aussi une histoire, une pièce politique et une tragédie romaine, Shakespeare, tout en utilisant abondamment des sources multiples, rivalise de virtuosité avec Plutarque, Robert Garnier, Samuel Daniel, et bien d'autres. La passion sans bornes de Marc

${ }^{141}$ Anthony and Cleopatra, éd. Michael Neill, Oxford, Oxford University Press, coll. World's Classics, 1994. Tout en adoptant cette édition, remarquable à beaucoup d'égards, je ne retiendrai pas cependant la graphie « Anthony » pour conserver l'orthographe habituelle du nom. 
Antoine pour la reine d'Égypte se déploie en effet dans le cadre d'un long et vaste affrontement politique entre les triumvirs, dont l'enjeu est la conquête du pouvoir et du monde ( «a drama of love is played out against the epic sweep of history", comme le dit bien David Bevington $)^{142}$, le tout sur une toile de fond cosmique et apocalyptique (« Let Rome in Tiber melt », I.i..35 ; « Melt Egypt into Nile », II.v.79). Le dramaturge jacobéen brosse une de ses fresques les plus ambitieuses, inspirée en partie du Tamburlaine de Marlowe, qu'il situe à un moment de crise dans l'histoire de Rome, lorsque la République finissante, encore hantée par la nostalgie de ses grandes figures héroïques, est sur le point d'accoucher de l'Empire et de la Paix Universelle chère à Octave César qui sera couronné sous le nom d'Auguste. Un ordre naissant se profile derrière le spectacle d'une décadence égyptienne qui est autant un contre-modèle qu'une source de fascination face à la tempérance et à la mesure romaines. Comme le dit Menas à Pompée qui, en homme honnête et scrupuleux, en républicain de l'ancien temps, se refusera à saisir l'occasion unique qui lui est offerte au moment de la bacchanale qui se déroule sur sa galère :

Thou art, if thou dar'st be, the earthly Jove :

Whate'er the ocean pales, or sky inclips,

Is thine, if thou wilt ha't.

(II.vii.68-70)

Pris, selon Plutarque, entre leur pacte de la vie inimitable ( « amimetobion ») et leur pacte de mort («synapothanumenon »), les amants du Nil oscillent dans la pièce entre l'ordure et l'or, entre le baiser de feu du dieu soleil et la fange fertile symbolisée par le scarabée hiéroglyphe ( « beetle », III.ii.20) au cœur de la magie quasi-alchimique de la fertilité et du renouveau obtenus par l'entremise des inondations périodiques du fleuve :

[...] they take the flow o'th'Nile

By certain scales i'th'pyramid; they know

By th'height, the lowness, or the mean, if dearth

Or foison follow. The higher Nilus swells,

The more it promises: as it ebbs, the seedsman

Upon the slime and ooze scatters his grain,

And shortly comes to harvest.

(II.vii.17-23)

${ }^{142}$ Antony and Cleopatra, éd. David Bevington, Cambridge, Cambridge University Press, 1990, p. 7 . 
Le phénomène bien connu des crues du Nil, souvent décrit par les Anciens (Hérodote, Ovide, Pline l'Ancien), donne ici du sens à ce régime de l'intempérance où s'exprime éminemment le style asiatique, c'est-à-dire un style hyperbolique, histrionique et extravagant. Selon David Bevington,

Egypt is accused of the very excesses that were objected to in the 'Asian' style: of being as it were inflatus (blown up), solutus (dissolved), tumens (swollen), superfluens, redundans, enervis (idle), inanus (empty) $)^{143}$

Dans la pièce, l'excès affecte aussi bien un langage tumescent, où toute exagération renvoie à un plus grand débordement pour mieux marquer ses propres limites, son impuissance à décrire, comme s'il était toujours en dessous ou en deçà de ce qu'il s'efforce de représenter. Le préfixe « o'er » revient à plusieurs reprises dans la pièce (« o'erpicturing », II.ii.207, " o'ercount », II.vi.26, ou encore « outwork », II.ii.207), on trouve des expressions comme «bounty overplus », IV.vi.21, et une foule de termes empruntant au registre de la munificence et de l'excellence. Les dialogues, les apostrophes et les gestes oscillent entre excès d'honneur et d'indignité, entre outrance et dérision, déréliction et démesure dans une série de scènes violemment contrastées où les personnages-titres sont tour à tour soumis aux filtres contradictoires de l'éloge et de l'injure, de la caricature et de la flatterie. Le langage de la pièce fonctionne au gré d'un mouvement de dépassement des limites en direction du noble, du beau, de l'héroïque, du sublime, mouvement qui suit ou précède un rabaissement vers l'anti-héroïque, vers le monde du pathétique, du sordide (ce qu'Enobarbus appelle « my turpitude », IV.vi.32), de la laideur et de la puanteur, qui touche au grotesque et parfois à l'insoutenable. L'excès fonctionne dans les deux sens, vers le haut comme vers le bas, dans un rythme effréné de montagnes russes qui donne un peu le vertige. C'est un régime en accordéon, où la réalité, l'histoire et les principaux personnages sont observés alternativement par l'un et l'autre bouts de la lorgnette, tantôt comme un théâtre où s'affrontent des demi-dieux et des géants, tantôt comme un microcosme un peu ridicule où s'agitent les marionnettes dérisoires que sont le «strumpet's fool » et la «foul Egyptian » (IV.xiii.10). Paradoxalement, le guerrier vaincu et déchu se

143 David Bevington, op. cit, p. 39. 
voit projeter dans les étoiles grâce à l'apothéose du rêve de Cléopâtre à la fin, alors que la reine reste, quant à elle, une figure changeante et mystérieuse, caractérisée par son « infinite variety » et qui ne se laisse fixer dans aucune image ni aucun signifiant pour mourir au milieu d'une phrase inachevée...

\section{$I$}

Comme l'explique Rosalie Colie dans des analyses qui sont reprises par Janet Adelman, David Bevington et Michael Neill, la vision de l'excès dans Antony and Cleopatra repose principalement sur le recours massif à l'hyperbole, figure que Puttenham décrit comme «the overreacher $[. .$.$] or loud lyer { }^{144} »$ :

It is the strength, the vividness, the vigor of excess which this play presents, examines, criticizes and ultimately [...] confirms, in a language of hyperbole built to match the size and scope of the subject [...] By sinking the notions associated with the Asiatic style back into life itself, in the play's dramatic action he can examine and assess both the style and the style of life in terms of each other, and to see them as one ${ }^{145}$.

À côté de l'hyperbole, on remarque aussi un recours fréquent à ce qu'on pourrait appeler les marqueurs de l'excès, des effets qui contribuent à donner de l'ampleur, de l'étoffe, de l'allant au discours. Il y a tout d'abord des adjectifs comme "infinite », " eternal/eternity », mais aussi des multiplicateurs comme « Ten thousand harms» (I.ii.129), « a million », « Being barbered ten times o'er » (II.ii.31), " the sevenfold shield of Ajax» (IV.xv.38), " his soldiership / Is twice the other twain » (II.i.34-5), qui jouent sur le quantitatif et sur l'effet d'accroissement ainsi obtenu. L'exemple le plus frappant est sans doute le qualificatif utilisé par Philo pour désigner le général quand il l'appelle « the triple pillar of the world » (I.i.12), d'un effet bien différent, même si le sens est le même, de la remarque facétieuse que fait Enobarbus à propos du serviteur qui emporte Lepidus ivre mort : «A bears / The third part of the world, man : seest not?» (II.vii.87-8). «Triple pillar» donne au départ une impression de puissance, encore renforcée par l'allitération

144 George Puttenham, The Arte of English Poesie (1589), Facsimile Reproduction, The Kent State University Press (1970), " Hiperbole. Or the Over reacher, otherwise called the loud lyer », p. 202.

145 Rosalie Colie, "Antony and Cleopatra: The Significance of Style », Shakespeare's Living Art, Princeton, New Jersey, Princeton University Press, 1974, p. 207. 
qui permet d'établir un contraste saisissant avec le vers suivant, "strumpet's fool ». D'ailleurs, il y a sans doute un lien à établir entre «triple pillar » et l'appellation de «triple-turned whore » (IV.xiii.13), dont Marc Antoine affublera Cléopâtre dans un moment de fureur jalouse. L'hypertrophie flatteuse se renforce ici au contact de son contraire qu'est l'injure pour former avec elle un couple paradoxal. Ces excès verbaux se retrouvent aussi dans les comparaisons avec les dieux ou les héros du panthéon mythologique et de l'épopée (Mars, Hercule, Ajax), par exemple lorsque Cléopâtre désigne son amant comme « this Herculean Roman » (I.iii.84). La taille d'ailleurs doit sans cesse se mesurer. Tantôt Marc Antoine n'est plus ce qu'il était, tantôt il est à nouveau égal à lui-même : «He comes too short of that great property / Which still should go with Antony» (I.i.59), image qui ouvre naturellement la voie à maints sous-entendus grivois :

CLEOPATRA. And when good will is showed, though't come too short, The actor may plead pardon.
(II.v.9-10)

Par ailleurs, Cléopâtre qui aime à s'emparer de la grande épée d'Antoine, lui déclare tout de go à la scène iii du premier acte : " I would I had thy inches... » (I.iii.39). Dans le même registre, après avoir dit envier le cheval qui porte le poids de son amant ( $\mathrm{O}$ happy horse, to bear the weight of Antony », I.v.21), elle reviendra à cette idée, cette fois de façon beaucoup plus concrète quand il s'agira de le hisser en haut du monument alors qu'il agonise :

Here's sport indeed! How heavy weighs my lord! Our strength is all gone into heavinessThat makes the weight...

(IV.xvi.34-6)

Cette façon qu'a Shakespeare de relier le désir à la mort lui permet de dire quelque chose de la sexualité féminine en associant l'hyperbole érotique et le monde du fantasme à un jeu de mots sur la notion de pesanteur qui, à travers le poids du corps d'Antoine, évoque directement et de façon très physique la lourdeur du chagrin ( " heaviness »). L'excès se résorbe ici en un humour macabre, à l'instar des bons mots de Mercutio frappé à mort au cours du duel avec Tybalt, et qui est bien une forme de politesse du désespoir. L'émotion surgit au détour d'une figure intériorisée de l'excès qui permet de traduire l'intensité de la douleur ressentie par la reine. 
Les va-et-vient incessants des messagers, les commentaires croisés qui multiplient les points de vue sur les personnages-titres et agissent comme un kaléidoscope, font enfler la rumeur, ce « common liar» (I.i.62) qui désigne aussi bien le mentir-vrai du qu'en-dira-t-on que la « marie couche toi là » d'Alexandrie, celle dont Enobarbus nous dit seulement que « she did lie / In her pavilion » (II.ii.206). Ce n'est pas l'un des moindres paradoxes de cette pièce où les voix du grotesque et du sublime semblent quasiment interchangeables.

Car on trouverait sans peine beaucoup d'autres exemples de ces tropes de la multiplication dans Antony and Cleopatra. Le domaine le plus riche et le plus récurrent, qui permet aussi de réunir les multiples facettes de l'excès dans la pièce, a trait à ce qui concerne le monde de l'appétit, de la table et de la boisson.

\section{II}

Il s'agit là d'une thématique sur laquelle Plutarque insiste, notamment quand il dépeint l'arbitraire du Triumvirat :

Now the government of these Triumviri grew odious and hateful to the Romans, for divers respects. But they most blamed Antonius, because he, being elder than Caesar and of more power and force than Lepidus, gave himself again to his former riot and excess when he left to deal in the affaires of the commonwealth. But, setting aside the ill name he had for his insolency, he was yet much more hated in respect of the house he dwelt in, the which was the house of Pompey the Great, a man as famous for his temperance, modesty, and civil life, as for his three triumphs. For it grieved them to see the gates commonly shut against the captains, magistrates of the city, and also ambassadors of strange nations, which were sometimes thrust from the gate with violence; and that the house within was full of tumblers, antic dancers, jugglers, players, jesters, and drunkards, quaffing and guzzling $[\ldots]^{146}$.

Dans la pièce, Pompée décrit un Marc Antoine enchaîné par les sens à la table et au lit de Cléopâtre :

Mark Antony

In Egypt sits at dinner, and will make

No wars without doors...

(II.i.11-13)

\footnotetext{
${ }^{146}$ Cité par Michael Neill, op. cit., Appendix A, p. 330.
} 
Et, un peu plus loin, il montre un général entièrement soumis aux caprices de la nouvelle Circé du Nil :

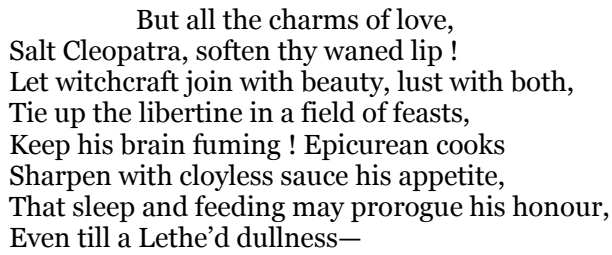

L'indolence et la mollesse sont ici rendues par la succession des « $\mathrm{i}$ » longs ([i :] ( « sleep », « feeding », « Lethe »). Quant aux images, elles constituent autant de variantes sur le thème biblique des « fleshpots of Egypt » (Exodus xvi.3) ainsi que sur le mythe de l'enchanteresse qui place les hommes sous sa coupe avant de les transformer en pourceaux, thème à la fois homérique et moderne puisqu'il est repris par Spenser dans The Faerie Queene, avant de devenir l'une des constantes des masques donnés à la cour des Stuarts. En ce début de XVII ${ }^{\mathrm{e}}$ siècle, au moment où se noue la collaboration entre le poète Ben Jonson et l'architecte Inigo Jones, le combat traditionnel entre Gula et Tempérance au sein de la Moralité prend désormais des allures somptueusement visuelles, grâce aux décors à l'italienne, aux rythmes de la danse et aux accents d'une musique raffinée. Celle que Pompée nomme «salt Cleopatra » parle elle-même de sa jeunesse comme de ses «salad days» (I.v.73) tandis qu'on l'évoque par les termes plus vulgaires de « dish » ou de « morsel » :

ANTHONY. I found you as a morsel, cold upon

Dead Caesar's trencher-nay, you were a fragment

Of Gnaeius Pompey's-besides what hotter hours,

Unregistered in vulgar fame, you have

Luxuriously picked out...

(III.xiii.117-21)

Comme l'indique malicieusement Enobarbus, l'Égypte est devenue pour Marc Antoine un vaste théâtre de l'appétit, où la magie de la reine consiste à ne jamais le rassasier :

Other women cloy

The appetites they feed, but she makes hungry

Where most she satisfies..

（II.ii.243-45） 
Celui que Pompée surnomme «the ne'er lust-wearied Antony» (II.i.38), et que Cléopâtre semble soumettre à ce qui ressemble plus à des délices qu'au supplice de Tantale, parait en effet voué à une forme de boulimie illimitée. Ces excès déjà légendaires de la vie égyptienne, que Cléopâtre appelle «our Alexandrian revels», v.ii.218), sont directement évoqués par Enobarbus dans le bref dialogue qui précède le récit de l'apparition de Cléopâtre sur le fleuve Cydnus :

MECENAS. We have cause to be glad that matters are so well digested. You stayed well by't in Egypt.

ENOBARBUS. Ay, sir, we did sleep day out of countenance, and made the night light with drinking.

MECENAS. Eight wild boars roasted whole at a breakfast, and but twelve persons there-is this true?

ENOBARBUS. This was but as a fly by an eagle. We had much more monstrous matter of feast, which worthily deserved noting. (II.ii.182-90)

De tels propos sont visiblement marqués à l'aune de la traditionnelle vantardise du voyageur, mais on voit aussi se mettre en marche une logique de la surenchère, que l'on retrouvera dans la description de Cléopâtre (avec des expressions comme " It beggared all description ", v. 205, ou « O'er picturing that Venus where we see / The fancy outwork nature », v. 207-8). Il s'agit de constamment changer d'échelle : «Eight wild boars... but twelve persons there », soit deux tiers de sanglier par personne au petit déjeuner, ce qui, comparé au " reste », qui n'est bien entendu pas décrit, représente la même différence que celle qui existe entre la taille d'une mouche et celle d'un aigle... Le curseur de l'excès, qui prend un tour visuel et quantitatif pour mieux mesurer, fera ensuite place à l'appel à l'imagination et aux ressources quasi-infinies de l'art par rapport à la nature ${ }^{147}$. Ainsi, toute représentation de l'excès dans la pièce donne à entendre, sinon à voir, un excès plus grand encore (" past the size », v.ii.97). De même, après l'évacuation de Lépide, alors que la fête bat son plein, Pompée déclare « This is not yet an Alexandrian feast » (II.vii.93). Le thème de l'excès est donc présenté comme ce qui ne cesse de dépasser, de déborder, le cadre fixé au départ («o'erflows the measure », I.i.2), ou ce qui est

\footnotetext{
147 «It's past the size of dreaming. Nature wants stuff To vie strange forms with Fancy, yet t'imagine An Antony were nature's piece 'gainst Fancy, Condemning shadows quite » (v.ii.97-100).
} 
encore loin d'atteindre ce qu'on attend d'elle, un peu comme le Marc Antoine «égyptien » est, aux yeux des Romains, désormais loin d'approcher l'homme d'exception qu'il fut un temps.

Mais, à côté du portrait de l'Antoine boulimique et dipsomane que dressent Pompée et Enobarbus, Octave garde, lui, la nostalgie d'un général anorexique, véritable champion de jeûne, héros à l'ancienne capable de survivre dans des conditions extrêmes :

When thou once

Was beaten from Modena, where thou slew'st

Hirtius and Pansa, consuls, at thy heel

Did Famine follow, whom thou fought'st against-

Though daintily brought up-with patience more

Than savages could suffer. Thou didst drink

The stale of horses, and the gilded puddle

Which beasts would cough at [...]

It is reported thou didst eat strange flesh

Which some did die to look on..

(I.iv.56-68)

César s'attache en effet à l'excès inverse dans ce discours où il s'adresse directement à Marc Antoine comme pour l'exhorter à se ressaisir, et il souligne avec force ces images en creux ou en négatif qui montrent un homme aux antipodes de ce qu'il est devenu en s'abandonnant corps et âme à l'influence voluptueuse et délétère de ce que Pompée nomme " [his] fine Egyptian cookery » (II.vi.64), sans oublier de rappeler au passage un précédent célèbre, «I have heard that Julius Caesar / Grew fast with feasting there » (II.vi.65-6). La rencontre de Marc Antoine et de Cléopâtre s'est d'ailleurs nouée autour d'une invitation à souper qui a vite tourné à l'avantage de la reine :

Upon her landing, Anthony sent to her,

Invited her to supper. She replied,

It should be better he became her guest,

Which she entreated. Our courteous Antony,

Whom ne'er the word of 'no' woman heard speak,

Being barbered ten times o'er, goes to the feast,

And for his ordinary pays his heart

For what his eyes eat only.

(II.ii.226-33)

Dans cette présentation malicieuse, Enobarbus, sans totalement tomber dans le piège des clichés misogynes qu'il ne prend par ailleurs pas grand peine à éviter, s'efforce en réalité de mettre en valeur le singulier pouvoir de Cléopâtre qui paraît reposer sur une forme de 
présence invisible ( «she did lie / In her pavilion... », II.ii.205-6). Elle incarne une véritable emprise, voire un empire, du négatif, où l'on pourra voir une illustration de la «negative capability » chère à John Keats ${ }^{8}$.

\section{III}

Cléopâtre, c'est en effet l'excès en creux qui émane de sa personne, qui fascine ses interlocuteurs comme ses amants jusqu'à ce qu'ils en soient comme vidés de l'intérieur comme le remarque Marc Antoine dans un accès de rage :

Betrayed I am.

O this false soul of Egypt! this grave charm,

Whose eye becked forth my wars and called them home,

Whose bosom was my crownet, my chief end,

Like a right gypsy hath at fast and loose

Beguiled me to the very heart of loss.

(IV.xiii.24-9)

Cléopâtre se confond avec l'image de l'escamoteuse, celle dont l'Hercule romain pensait ne faire qu'une bouchée au banquet des sens où il la convie, alors qu'en lui retournant la politesse (incidemment, la double négation du vers "Whom ne'er the word of 'no' woman heard speak » constitue une hyperbole galante que la reine va s'amuser à mettre à mal), c'est elle qui va bel et bien le vider de sa substance. Dans la scène $\mathrm{v}$ de l'acte II, elle rappelle en riant aux éclats tous les bons tours qu'elle a joués à son amant, appâté par l'hameçon de ses courbes tout comme les beaux poissons du Nil sont pris avec son hameçon recourbé, ou encore habillé en femme et dépouillé de sa grande épée tandis qu'il cuve son vin :

\footnotetext{
I laughed him out of patience, and that night I laughed him into patience, and next morn, Ere the ninth hour, I drank him to his bed.

Then put my tires and mantles on him, whilst I wore his sword Philippan.
}

C'est cette absence de limites, ce goût de l'excès, de la provocation et de la transgression, qui la conduisent à faire de ses amours des Saturnales permanentes, qui inquiètent le monde romain. Ce dernier, sans être par principe hostile à la fête, ne souhaite cependant pas qu'elle devienne la règle. Dès lors il n'est guère surprenant qu'il paraisse 
préférer une Octavie sobre, froide et ennuyeuse (II.vi.121-22 ; v.ii.54-5) à l'intempérance (III.xiii.121-23) et à la tyrannie capricieuse de Cléopâtre. Mécène déclare en effet à Octavie en présence d'Agrippa :

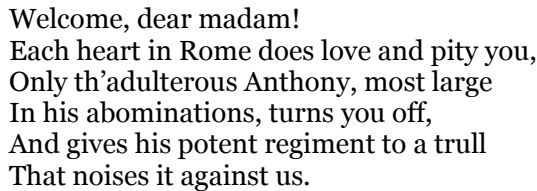$$
\text { (III.vi.92-7) }
$$

Marc Antoine, lui, bien qu'il soit totalement subjugué par celle qu'il appelle tour à tour la fée, la magicienne ou la sorcière, éprouve dans le même temps un sentiment contradictoire et confus, l'impression d'être à la fois dissolu et dissous sous l'effet d'une passion pour une femme dangereuse qui le pousse constamment au-delà de lui-même dans une course folle où il faut des libéralités toujours plus grandes, plus de fastes, de dépenses somptuaires et de débauches. Si l'Égypte est indiscutablement le lieu de son plaisir (« I'th'East my pleasure lies », II.iii.40), il se reproche simultanément une vie émolliente et oisive, justifiant ainsi rétrospectivement le reproche de Demetrius puisqu'il reprend à son compte le mot « dotage » :

These strong Egyptian fetters I must break

Or lose myself in dotage [...]

I must from this enchanting queen break off:

Ten thousand harms, more than the ills I know,

My idleness doth hatch..

(I.ii.116-30)

Enobarbus, en quittant son maître, fait le constat désenchanté que le vaisseau amiral fait eau de toutes parts :

Sir, sir, thou art so leaky

That we must leave thee to thy sinking...

(III.xiii.64-65)

Octave, quant à lui, avait dès le début, fait de Marc Antoine une sorte de tonneau des Danaïdes :

If he filled

His vacancy with his voluptuousness,

Full surfeits and the dryness of his bones

Call on him for't

(I.iv.25-7)

Effectivement, le général humilié reconnaît sa défaite à la bataille d'Actium en ces termes : «Authority melts from me » (III.xiii.90). Puis, 
enfin apaisé, sinon résigné, il voit dans cet effacement de l'identité une sorte de loi naturelle plus ou moins inévitable :

That which is now a horse, even with a thought

The rack dislimns, and makes it indistinct

As water is in water $[\ldots]$

here I am Antony

Yet cannot hold this visible shape...

(IV.xV.9-14)

Contrairement à son amant, définitivement marqué par la culture romaine, par le sens de l'honneur et du devoir qu'il ne réussit jamais à faire disparaître de son horizon personnel, Cléopâtre se sent tout à fait dans son élément quand il s'agit des eaux ondoyantes et débordantes du Nil à qui elle s'identifie. Comme l'écrit Kim Hall :

[...] the Nile runs throughout Antony and Cleopatra as a sign of overwhelming sexuality and social disorder and associates Cleopatra with the kind of overflow and excess characteristic of the female grotesque [...] More than a wishing-away of worldly cares or a sign of Egyptian dispersal of symbols of order and measurement, the metaphors of excess bespeak an anxiety striking directly at the heart of Europe's primal fear: loss of identity in measureless expansion [...] although it is true that "the luxury and feasting of the Egyptian court image a natural plenty which is curbed by no Roman temperance", this natural plenitude, so seductive to a modern audience, is precisely what would have been threatening to a Europe struggling to control its own countrymen loosed into a foreign world of plenty ${ }^{148}$.

Car les excès de Cléopâtre sont directement liés à l'hydrographie sacrée du fleuve et au culte qui lui était voué, de sorte qu'elle réconcilie les contraires en faisant de la perte un gain et de la destruction une promesse de renouveau et de fertilité :

For vilest things

Become themselves in her, that the holy priests

Bless her when she is riggish.

(II.ii.245-47)

Dans cette perspective, loin d'être simple intempérance, abus ou transgression, l'excès devient la condition de l'accès à un nouveau mode d'être, où il faut mourir à soi pour pouvoir renaitre. Cette interprétation revient effectivement à plusieurs reprises dans la pièce où l'on nous explique par tout un ensemble de paradoxes qui ne sont

${ }^{148}$ Kim Hall, Things of Darkness. Economies of Race and Gender in Early Modern England, Ithaca et Londres, Cornell University Press, 1995, p. 157. 
pas des formules isolées qu'il faut quitter pour suivre, trahir pour aimer, et perdre pour gagner. Selon Barbara Bono, ce sont là des éléments qui s'appuient sur le mythe d'Isis que Cléopâtre reprendrait à son compte :

The greatest harvest comes from seemingly greatest destruction; generation results from the warming action of sun on slime. The Egyptians rejoice in this natural discordia concors, and include in it the worship of an earthly sexuality [...] Cleopatra, identifying with Isis, is proud and unselfconscious about her sexuality, delighting in her multiple Roman conquests and indulging in erotic musings before her court. Her sexuality is sacred, expressive of the fertility of her country. But her erotic energy, like the cycles of flood and harvest, has its terrifying side, as when, in a jealous rage, she wishes "infectious pestilence" on the messenger from Rome [...] Because fertility in Egypt is achieved through such destructive phases, not in denial of them, Cleopatra freely enacts such excesses as catharsis ${ }^{149}$.

On trouve un équivalent de cette coïncidence des contraires dans la double vocation d'Antoine qui, selon les dires de Demetrius, «is become the bellows and the fan / To cool a gypsy's lust» (I.i.9-10), affirmation étonnante qui trouvera un écho direct dans la description par Enobarbus des cupidons souriants qui entourent Cléopâtre sur sa barge :

On each side her

Stood pretty, dimpled boys, like smiling Cupids, With divers-coloured fans, whose wind did seem To glow the delicate cheeks which they did cool, And what they undid did.

(II.ii.208-12)

En réchauffant et en rafraîchissant simultanément les joues de la reine, les éventails-soufflets jouent un peu le rôle de corrélat objectif de l'excès qui, dans la pièce, se trouve immédiatement refroidi par l'ironie ou le sarcasme. Mais le schéma inverse est également utilisé comme le montrent les stichomythies du début sur la possibilité ou non de quantifier l'amour :

ClEOPATRA. If it be love indeed, tell me how much.

ANTONY. There's beggary in the love that can be reckoned.

CLEOPATRA. I'll set a bourn how far to be beloved.

149 Barbara J. Bono, Literary Transvaluation. From Vergilian Epic to Shakespearean Tragicomedy, Berkeley, Los Angeles et Londres, University of California Press, 1984 p. 205 . 
ANTONY. Then must thou needs find out new heaven, new earth.

$$
\text { (I.i.14-17) }
$$

Ces déclarations grandiloquentes du Romain qui ne sont apparemment là qu'à titre d'esquive ou de dérobade pour préparer sa sortie et amadouer la reine, seront reprises à la lettre par cette dernière dans le blason cosmique qu'elle consacrera à son héros en guise d'ultime déclaration d'amour :

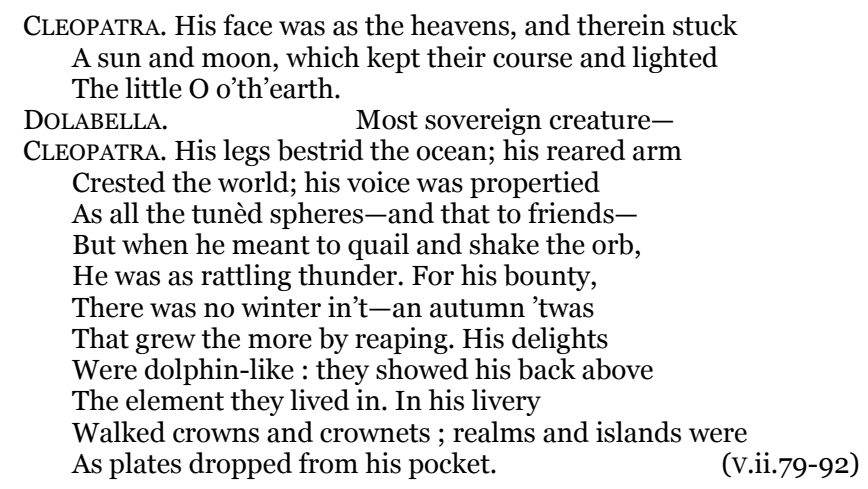

Cléopâtre recourt au registre du sublime en composant ce blason masculin à la dimension du cosmos, où les parties du corps aimé, dans ce qu'il a de plus physique (et l'amour dans la pièce est d'abord et avant tout marqué par la sensualité et ses fortes composantes érotiques), se trouvent projetées dans le champ des étoiles et des planètes tandis que sa voix s'identifie au grondement du tonnerre. C'est là sans doute la contrepartie positive du désastre annoncé de la chute du héros: «When my good stars, that were my former guides, / Have empty left their orbs, and shot their fires / Into th'abysm of hell ", (III.xiii.14648), ou encore "Alack, our terrene moon / Is now eclipsed and it portends alone / The fall of Antony » (III.xiii.154-56). Mais ce qui paraît remarquable, c'est moins cette apothéose que l'identification d'une source d'énergie renouvelable à l'infini, analogue à un principe de fertilité, à savoir l'éloge d'une générosité comparée à un cycle saisonnier sans hiver. Car là, en somme, réside la vraie justification de l'excès qui, en défaisant le héros, l'a paradoxalement « fait » (« what they undid did », II.ii.212), en lui permettant de devenir ce qu'il était. On pourrait encore dire qu'en le détruisant, l'excès lui donne accès à un 
mode d'être universel et éternel. Comme un bonheur ne vient jamais seul, cette démesure, qui relève somme toute d'une forme d'hybris comique et carnavalesque, se trouve miraculeusement transcrite dans la joie bondissante et rhapsodique des deux demi-vers allitératifs respectivement disposés en fin et en début de vers suivant: " His delights / Were dolphin-like » (v.ii.88-9). Désormais, Marc Antoine n'est plus l'un de ces petits poissons bariolés qu'on pêche dans les eaux du Nil, il est devenu le roi de l'élément marin dans lequel il s'était prétendument dissous après sa défaite dans la bataille navale contre César.

Cette vision noble et généreuse de l'amour sublimé et poursuivi au-delà de la mort s'oppose terme à terme à l'excès inverse de sa réduction caricaturale dans la pornographie romaine qui braque le projecteur sur l'obscénité et la vulgarité par le biais du travestissement grotesque et d'une sorte de voyeurisme haineux :

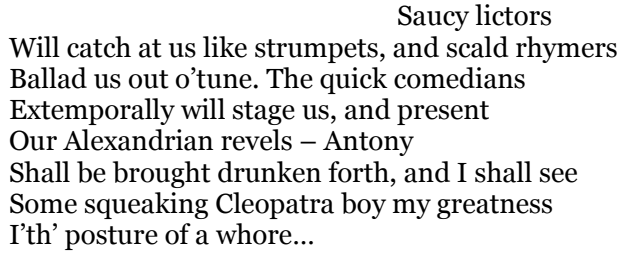

En choisissant ce qu'elle appelle «the high Roman fashion » (IV.xvi.88), et donc la mort volontaire, pour échapper à l'humiliation et à la caricature, Cléopâtre confirme que l'excès peut ouvrir la voie à un certain héroïsme. La mise en scène très théâtrale de son suicide, dont l'instrument plongera un temps César dans la perplexité, associe la surprise et la ruse avec le sens du mystère, tout en donnant à voir dans ce sacrifice un mariage mystique, une maternité pervertie (l'aspic mordant le sein est une image dévoyée de la Vierge à l'enfant), une manière d'ultime jouissance.

Ce bref aperçu ne prend en compte que quelques unes des nombreuses figures de l'excès dans Antony and Cleopatra. Il aurait fallu parler aussi de la question de la générosité et de la munificence, où la question de l'excès semble liée à une philosophie du don, ou potlatch, dont Shakespeare montrera ensuite les limites dans Timon of Athens. 
La musique, si importante dans la pièce, a elle aussi ses excès, qu'il s'agisse du son des flûtes qui rythment les mouvements des rames argentées de la barge de Cléopâtre (II.ii.202) ou de l'opposition entre, d'un côté, le tintamarre assourdissant qui réunit le ciel et la terre dans une sorte d'ivresse quasi-dionysiaque, dans une énergie dépensée jusqu'à la folie (II.vii.108-11 ; Iv.ix.35-40) et, de l'autre, l'étrange scène où l'on entend une musique venue d'ailleurs, un air plein de tristesse et de mélancolie joué par des hautbois et qui sert à signaler l'abandon de Marc Antoine par le dieu Hercule (Iv.iii.10-20).

Dans Antony and Cleopatra, les figures de l'excès servent à marquer les mensonges et les impasses du discours héroïque, dont les forfanteries (dans les hyperboles comme "I'll make Death love me » qui entraîne immédiatement le sarcasme d'Enobarbus " Now he'll outstare the lightning», III.xiii.193, 195, ou encore comme «I, that with my sword / Quartered the world, and o'er green Neptune's back / With ships made cities »..., Iv.xv.57-8) sont aussitôt démenties ou dénoncées comme autant de gesticulations d'impuissance. Ces figures se trouvent relayées par le truchement du théâtre de l'appétit et des tropes de la sexualité et de l'érotisme, où la force n'est plus du côté de celui qu'on croit. Elles servent à mettre en évidence le déchirement intérieur de Marc Antoine autant que la jubilation ou les déchaînements tragicomiques de Cléopâtre mais, par le jeu quasidialectique du renversement des contraires qui opère tout au long de l'œuvre, elles aboutissent à réhabiliter celui qui était critiqué pour sa mollesse et ses atermoiements. Bien que vaincu à la fin, il n'est nullement déchu mais bien au contraire propulsé au firmament pour devenir, aux côtés de sa reine, le véritable héros d'une histoire qui finit par marginaliser celui qui en est pourtant le grand vainqueur aux yeux du monde, à savoir Octave César. Les figures de l'excès participent donc pleinement de cette hyperontologie paradoxale bien mise en valeur par H. Fawkner dans l'ouvrage essentiel qu'il a consacré à cette tragédie ${ }^{150}$.

François LAROQUE

${ }^{150}$ H. W. Fawkner, Shakespeare's Hyperontology. Antony and Cleopatra, Londres et Toronto, Associated University Press, 1990. 